\title{
BMJ Open Prewarming i-gel laryngeal mask for mechanical ventilation: a meta-analysis of randomised control trials and trial sequential analysis
}

\author{
Jianqiao Zheng (D,${ }^{1}$ Li Du, ${ }^{2}$ Jia Wang, ${ }^{1}$ Lu Zhang, ${ }^{1}$ Guo Chen ${ }^{1}$
}

To cite: Zheng J, Du L, Wang J, et al. Prewarming i-gel laryngeal mask for mechanical ventilation: a meta-analysis of randomised control trials and trial sequential analysis. BMJ Open 2021;11:e045461. doi:10.1136/ bmjopen-2020-045461

- Prepublication history and additional supplemental material for this paper are available online. To view these files, please visit the journal online. (http://dx.doi.org/10.1136/ bmjopen-2020-045461).

Received 01 October 2020 Accepted 15 July 2021

Check for updates

(C) Author(s) (or their employer(s)) 2021. Re-use permitted under CC BY-NC. No commercial re-use. See rights and permissions. Published by BMJ.

${ }^{1}$ Department of Anesthesiology, Sichuan University West China Hospital, Chengdu, China ${ }^{2}$ Department of Anesthesiology, Sichuan Cancer Hospital and Research Institute, Chengdu, China

Correspondence to

Professor Guo Chen;

Anesthesiology_SCU@163.com

\section{ABSTRACT}

Objective To systematically evaluate the efficiency of prewarming i-gel laryngeal mask for mechanical ventilation by meta-analysis and trial sequential analysis. Design Systematic review and meta-analysis.

Data sources Cochrane library, Web of Science, Ovid Medline and PubMed were searched from their inception to 31 August 2020. Only articles published in English language were considered.

Methods Randomised controlled trials (RCTs) comparing the efficiency of prewarming i-gel laryngeal mask versus keeping it at room temperature for mechanical ventilation were included. Primary outcome was sealing pressure immediately after successful ventilation. Secondary outcomes were the first-attempt insertion success rate and the incidence of postoperative pharyngeal pain. Two authors independently selected studies. Quality analysis was performed using the modified Jadad Scale. Trial sequential analysis (TSA) was used to control risk of random errors. Sensitivity analysis was done to assess the effect of a single study on the pooled estimates. Publication bias was assessed by funnel plots and Egger's regression test.

Results Four RCTs comprising 374 patients were included. The results of meta-analysis showed that compared with the control group, prewarming i-gel laryngeal mask provides a higher sealing pressure immediately after successful ventilation (mean difference $2.19 \mathrm{~cm} \mathrm{H}_{2} 0 ; 95 \% \mathrm{Cl}$ (1.17 to 3.21); $p<0.0001$; high quality), with firm evidence from TSA and lower publication bias $(p=0.7372)$. No significant difference was observed in the first-attempt insertion success rate (relative ratio (RR) $1.06 ; 95 \% \mathrm{Cl}$ (1.00 to 1.12); $p=0.07$; high quality) with lower publication bias $(p=0.1378)$. The TSA indicating further trials are required. No significant difference was assessed in the incidence of postoperative pharyngeal pain (RR 1.0; $95 \% \mathrm{Cl}$ (0.14 to 6.90); $p=1.0$; high quality).

Conclusion Prewarming i-gel laryngeal mask provides higher sealing pressure compared with keeping it at room temperature. But prewarming i-gel laryngeal mask did not increase the first-attempt insertion success rate, nor did it decrease the incidence of postoperative pharyngeal pain.

\section{INTRODUCTION}

I-gel laryngeal mask (Intersurgical, Berkshire, UK) is a relatively new, non-inflatable,
Strengths and limitations of this study

- Language bias was potential possibility as the search strategy was limited to the English language only.

- The modified Jadad Scale was applied to assess the quality of literatures.

- Trial sequential analysis was used to assess the optimal sample size for the outcomes and control the risk of random errors.

- Funnel plots and Egger's regression test were used to assess the Publication bias.

- Limited number of studies with small sample were included may represent an important setback.

single-use, thermoplastic supraglottic airway device, which can be used as a safe and suitable alternative to endotracheal tube for elective surgeries in adult and paediatric patients. ${ }^{12}$ It has an integral bite block and a buccal cavity stabiliser which mirrors the oropharyngeal curvature of the patient and prevents malrotation. ${ }^{3}{ }^{4}$ The thermoplastic nature of the i-gel cuff will become soft and match the laryngeal anatomy better as it reaches body temperature after insertion. ${ }^{5}$

Sealing pressure is essential indicator for measurement of the successful placement, mechanical ventilation efficiency and prevention of aspiration. ${ }^{67}$ It is an indicator of how well a supraglottic device match the laryngeal anatomy during mechanical ventilation. ${ }^{8}$ Higher sealing pressure results from the closer contact between the cuff and the adjacent soft tissues. Previous reports have shown that i-gel cuff forms a more efficient seal around the larynx with a higher sealing pressure as the temperature rises to body temperature. ${ }^{9-11}$

Usually, i-gel laryngeal mask is preserved at room temperature before intubation. Compared with room temperature, prewarming i-gel laryngeal mask would enable 
the cuff to fit the pharyngeal structure more quickly. ${ }^{5}$ As the nature of thermoplastic material, prewarming laryngeal mask can increase the total successful insertion rate, shorten the insertion time and reduce the incidence of pharyngeal pain, which has been confirmed in clinical studies with relatively small sample..$^{512} 13$ So, if prewarming i-gel laryngeal mask could enable the cuff to fit the pharyngeal structure more quickly and provide better mechanical ventilation efficiency, it will be beneficial for intraoperative airway management and decrease the postoperative pharyngeal pain. But the melting point of i-gel cuff is above $200^{\circ} \mathrm{C}$, whether it will soften as it reaches body temperature after insertion remains controversial in clinical practice. ${ }^{14}$ So, the efficiency of prewarming i-gel laryngeal mask for mechanical ventilation remains unclear. ${ }^{15-17}$ This study aims to investigate the efficiency of prewarming i-gel laryngeal mask for mechanical ventilation by meta-analysis and trial sequential analysis (TSA).

\section{MATERIALS AND METHODS \\ Search strategy}

This meta-analysis was performed according to the Preferred Reporting Items for Systematic Reviews and Meta-Analyses guideline. ${ }^{18}$ According to the predetermined strategies, the relevant studies were obtained from Cochrane library, Web of Science, Ovid Medline and PubMed databases, with the searching keywords of (Prewarming OR Pre-warming OR Preheating OR Preheating) AND (i-gel OR i gel OR i-gel laryngeal mask airway) (search queries available in online supplemental appendix 1). Search strategy of PubMed (as an example) as follows:

\#1 "Laryngeal Masks"[Mesh] OR laryngeal mask airway*[Title/Abstract] OR laryngeal mask*[Title/ Abstract] OR aryngeal mask*[Title/Abstract] OR arynx mask*[Title/Abstract] OR LMA[Title/Abstract]

\#2 i-gel [Title/Abstract] OR igel [Title/Abstract] OR i gel [Title/Abstract]

\#3 \#1 OR \#2

\#4 random*[Title/Abstract] OR blind*[Title/Abstract] OR singleblind* [Title/Abstract] OR doubleblind* [Title/Abstract] OR trebleblind* [Title/Abstract] OR tripleblind*[Title/Abstract]

\#5 "Clinical Trials as Topic"[Mesh]

\#6 \#4 OR \#5

\#7 Prewarming [Title/Abstract] OR Pre-warming [Title/Abstract] OR Pre-heating [Title/Abstract] OR Pre-heating [Title/Abstract]

\#8 \#3 AND \#6 AND \#7

The search of the databases was performed from their inception to 31 August 2020 and was restricted to English language publications only. Manual retrieval was also performed for paper documents, and the references of related reviews and included studies were further screened to obtain more appropriate studies.

\section{Inclusion and exclusion criteria}

The inclusion criteria for studies analysed in this metaanalysis were as follows: (1) population-adult patients underwent mechanical ventilation in the supine position during general anaesthesia; (2) interventionprewarming i-gel laryngeal mask before insertion; (3) comparison-i-gel laryngeal mask keeping at room temperature before insertion; (4) outcome-sealing pressure immediately after successful ventilation as the primary outcome, first-attempt insertion success rate and the incidence of postoperative pharyngeal pain as the secondary outcome measures; (5) design-randomised control trials.

The following were exclusion criteria: (1) studies with incomplete, incorrect data or the research data could not be used for statistical analysis; (2) studies were abstracts from conferences, editorials, duplicate publications, letters, reviews and retrospective studies.

\section{Data extraction and quality assessment}

From the included trials, two authors independently extracted the following data: name of the first author, year of publication, participant characteristics and number, the prewarmed temperature of i-gel laryngeal mask and the room temperature of preserved laryngeal mask. The primary outcome of this review was sealing pressure immediately after successful ventilation. Additional outcomes such as the first-attempt insertion success rate and the incidence of postoperative pharyngeal pain were included as secondary outcomes. We also extracted numerical data from graphs using Adobe Photoshop as described by Gheibi $e t a l^{19}$

Two authors evaluated the risks of bias in the selected articles according to the Cochrane risk of bias assessment tool consisting of random sequence generation, allocation concealment, blinding of participants and personnel, blinding of outcome assessment, incomplete outcome date, selective reporting and other bias. ${ }^{20}$ Disagreement between investigators was resolved by consensus. The modified Jadad scale was applied to assess the quality of literatures (table 1). ${ }^{21}$ The overall quality of included studies was conducted in four aspects: randomisation (with score 0-2), allocation concealment (with score 0-2), blinding (with score 0-2), and withdrawals or dropouts (with score $0-1$ ). Scale scores range from 0 to 7 points, with higher scores indicating better quality. 1-3 were defined as low quality while $4-7$ were defined as high quality.

\section{Statistical analysis}

Analyses were performed using Review Manager V.5.4 (Rev Man, Cochrane Collaboration, Oxford, UK) and Stata/MP V.16.0 (Stata Corp). Group differences in dichotomous data were expressed as risk ratio (RR) with a 95\% CI and group differences in continuous data as mean differences (MDs) with 95\% CIs. Heterogeneity was quantified using the $\mathrm{I}^{2}$ statistic, and $\mathrm{I}^{2}>50 \%$ indicated the presence of heterogeneity. ${ }^{18}$ If heterogeneity was significant, 
Table 1 The modified Jaded Scale

\begin{tabular}{|c|c|c|}
\hline Classification & Description & Score \\
\hline \multicolumn{3}{|l|}{ Randomisation } \\
\hline No & Semirandomised or quasi-randomised trials & 0 \\
\hline Yes & Random sequences produced by a computer or a random number table & 2 \\
\hline \multicolumn{3}{|c|}{ Allocation concealment } \\
\hline Unclear & Only use of a random number table or other random assignment scheme & 1 \\
\hline Yes & A method for assigning sequences without prediction & 2 \\
\hline \multicolumn{3}{|l|}{ Blinding } \\
\hline No & Use of double blindness without an appropriate method & 0 \\
\hline Unclear & Only mention of double blindness & 1 \\
\hline No & No description of withdrawal or dropouts & 0 \\
\hline Yes & A description of withdrawal or dropouts & 1 \\
\hline
\end{tabular}

the random-effect model was used. Otherwise, the fixedeffect model was used. $\mathrm{P}<0.05$ was considered statistically significant. Sensitivity analysis was done to assess the effect of a single study on the pooled estimates. Publication bias was assessed by visual judgement of the funnel plots asymmetry and more objectively through Egger's regression test. $^{22} 23$ The level of $\mathrm{p}<0.05$ was considered statistically significant and indicated potential publication bias.

\section{Trial sequential analysis}

TSA was performed to analysis the outcomes in order to calculate the required information size (RIS) and correct the risks of type I error and type II error. ${ }^{2425}$ TSA monitoring boundaries and RIS were both quantified. For continuous outcomes, the pooled meta-analysis estimated of included trials were used to estimate the anticipated $\mathrm{MD}$ and variance. The risk of type I error was maintained at $2.5 \%$ with a power of $90 \%$. It is hard to determine a clinically significant value of the $\mathrm{SD}$ and the variance in the sealing pressure. So, the MD and variance were defined by selecting the 'Low-bias Based' option in the TSA viewer. ${ }^{26}$ For dichotomous outcomes, RIS was calculated based on the proportion of participants with an outcome in the control group and relative risk reduction of $-20 \%$. The risk of type I error was maintained at $2.5 \%$ with a power of $90 \%$. TSA was performed using the TSA program V.0.9.5.10 Beta (http://www.ctu.dk/tsa).

\section{Patient and public involvement}

No patient involved.

\section{RESULTS}

\section{Characteristics of included studies}

A flow diagram of the literature search and reasons for exclusion are shown in figure 1. We analysed four trials $^{5}{ }^{15-17}$ involving 374 patients that met all the criteria (table 2).

\section{Quality of included studies}

The methodological bias of the eligible studies was presented in table 3. Random sequence generation was considered as low risk of bias in all included studies, and allocation concealment was described in three RCTs. ${ }^{515} 16$ All included studies were earned scores of $\geq 6$ for quality on the modified Jadad scale. The data demonstrate that our systematic review articles are of high quality (table 4).

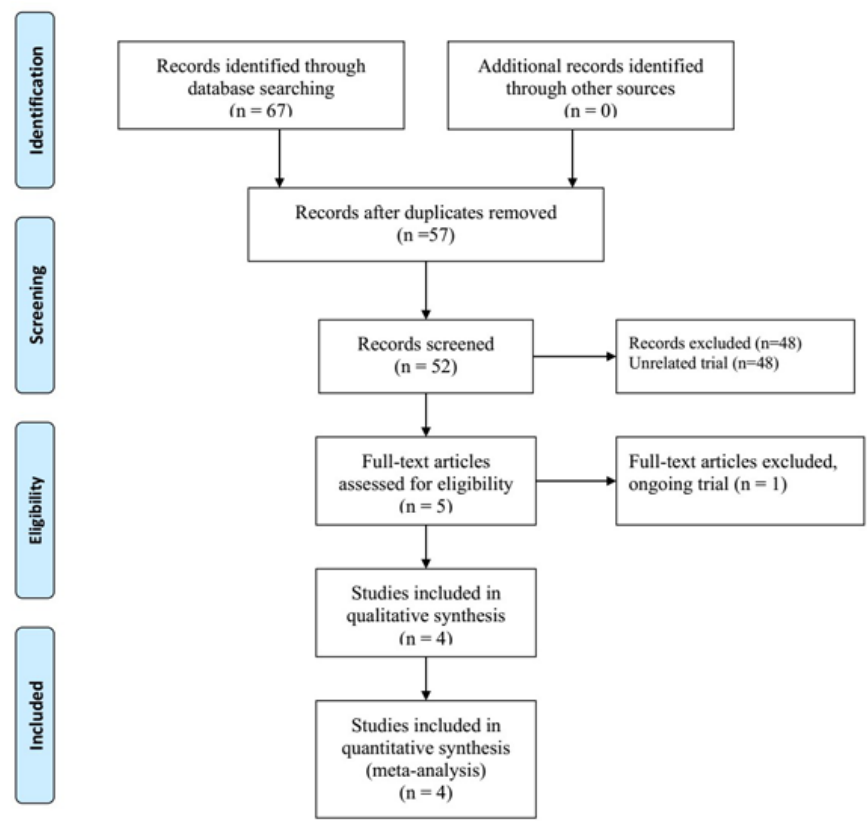

Figure 1 Flow chart of the trials included in the metaanalysis. 
Table 2 Characteristics of included studies

\begin{tabular}{|c|c|c|c|c|c|c|c|c|}
\hline Study & Year & Participants & Group & $\mathbf{N}$ & $\begin{array}{l}\text { Age, year } \\
(\mathrm{MD} \pm \mathrm{SD})\end{array}$ & $\begin{array}{l}\text { Gender } \\
(\mathrm{M} / \mathrm{F})\end{array}$ & $\begin{array}{l}\text { Intervention } \\
\text { (temperature before } \\
\text { insertion) }\end{array}$ & Outcomes \\
\hline \multirow[t]{2}{*}{$\begin{array}{l}\text { Komasawa } \\
\text { et } a l^{5}\end{array}$} & \multirow[t]{2}{*}{2014} & \multirow{2}{*}{$\begin{array}{l}\text { Adult patients } \\
\text { underwent general } \\
\text { anaesthesia in the } \\
\text { supine position }\end{array}$} & W group & 34 & $60 \pm 17$ & $21 / 13$ & $\begin{array}{l}\text { Prewarming to } 42^{\circ} \mathrm{C} \\
\text { for } 30 \mathrm{~min}\end{array}$ & $A B C$ \\
\hline & & & C group & 34 & $68 \pm 16$ & $20 / 14$ & $\begin{array}{l}\text { Room temperature } \\
\text { (approximately } 23^{\circ} \mathrm{C} \text { ) }\end{array}$ & \\
\hline $\begin{array}{l}\text { Nishiyama et } \\
a l^{15}\end{array}$ & 2012 & $\begin{array}{l}\text { Adult patients } \\
\text { underwent general } \\
\text { anaesthesia in the } \\
\text { supine position }\end{array}$ & C group & 86 & $50 \pm 14$ & $45 / 41$ & $\begin{array}{l}\text { Room temperature } \\
\text { (approximately } 20^{\circ} \mathrm{C} \text { ) }\end{array}$ & \\
\hline \multirow{2}{*}{$\begin{array}{l}\text { Komasawa } \\
\text { et } a l^{16}\end{array}$} & \multirow[t]{2}{*}{2015} & \multirow{2}{*}{$\begin{array}{l}\text { Adult patients } \\
\text { underwent general } \\
\text { anaesthesia in the } \\
\text { supine position }\end{array}$} & W group & 37 & $56.5 \pm 16.0$ & $18 / 19$ & Prewarming to $42^{\circ} \mathrm{C}$ & A B C \\
\hline & & & C group & 37 & $55.0 \pm 16.7$ & $22 / 15$ & Room temperature & \\
\hline Reddy et al ${ }^{17}$ & 2019 & $\begin{array}{l}\text { Adult patients } \\
\text { underwent general } \\
\text { anaesthesia in the } \\
\text { supine position or } \\
\text { lithotomy position }\end{array}$ & C group & 32 & $42.13 \pm 12.27$ & $20 / 12$ & $\begin{array}{l}\text { Room temperature } \\
\text { (approximately } 23^{\circ} \mathrm{C} \text { ) }\end{array}$ & \\
\hline
\end{tabular}

A: sealing pressure immediately after successful ventilation; B: intubation success at first attempt; C: incidence of postoperative pharyngeal pain.

C group, control group; F, female; M, male; N, number; W group, prewarming group.

Sealing pressure immediately after successful ventilation All RCTs reporting on the sealing pressure immediately after successful ventilation were included containing 185 patients in the prewarming group and 189 patients in the control group. ${ }^{515-17}$ The sealing pressure of the prewarming group was significantly higher than the control group (MD $2.19 \mathrm{~cm} \mathrm{H}_{2} \mathrm{O}$; $95 \% \mathrm{CI}, 1.17$ to 3.21 ; $\mathrm{p}<0.0001$; figure 2A). There was low heterogeneity $\left(\mathrm{I}^{2}=35 \%\right.$ and $\left.\mathrm{p}=0.42\right)$. In all included studies, only Komasawa et al did not use muscle relaxant in their research. ${ }^{16}$ So, we conducted sensitivity analyses. When this study is removed from the analysis, the combined result is similar to the original analysis and there is a significant improvement on the sealing pressure ( $\mathrm{MD} 2.24 \mathrm{~cm} \mathrm{H}_{2} \mathrm{O}, 95 \% \mathrm{CI}$ 1.14 to $3.33 ; \mathrm{p}<0.0001)$. Low heterogeneity was also indicated $\left(\mathrm{I}^{2}=35 \%\right.$ and $\left.\mathrm{p}=0.25\right)$.

The TSA revealed that the accrued information size $(\mathrm{n}=374)$ reached only $71.4 \%$ of the estimated RIS $(\mathrm{n}=524)$. But the cumulative $\mathrm{Z}$ score crossed the trial sequential monitoring boundary (figure 2B). So, TSA of pooled meta-analysis showed firm evidence for anticipated intervention effect.

\section{The first-attempt insertion success rate}

All RCTs on the first-attempt insertion success rate were included containing 185 patients in the prewarming group and 189 patients in the control group. ${ }^{5}{ }^{15-17}$ Data showed no significant difference in the first-attempt insertion success rate between the prewarming group and the control group (figure 3A): RR 1.06 (95\% CI 1.00 to $1.12 ; \mathrm{p}=0.07)$. There was low heterogeneity $\left(\mathrm{I}^{2}=35 \%\right.$ and $\mathrm{p}=0.20$ ). For this outcome, TSA shows that the RIS is 818 patients, which is larger than the current sample (374). The Z-curve did not cross the trial sequential monitoring boundary, which indicates that this effect remains uncertain and further trials are required (figure 3B).

\section{The incidence of postoperative pharyngeal pain}

Two articles on the incidence of postoperative throat pain were included containing 71 patients in the prewarming

Table 3 Risk of bias assessment

\begin{tabular}{|c|c|c|c|c|c|c|c|}
\hline Reference & $\begin{array}{l}\text { Random } \\
\text { sequence } \\
\text { generation }\end{array}$ & $\begin{array}{l}\text { Allocation } \\
\text { concealment }\end{array}$ & $\begin{array}{l}\text { Blinding of } \\
\text { participant } \\
\text { and personnel }\end{array}$ & $\begin{array}{l}\text { Blinding of } \\
\text { outcome } \\
\text { assessment }\end{array}$ & $\begin{array}{l}\text { Incomplete } \\
\text { outcome data }\end{array}$ & $\begin{array}{l}\text { Selective } \\
\text { reporting }\end{array}$ & $\begin{array}{l}\text { Other } \\
\text { bias }\end{array}$ \\
\hline Komasawa et $a l^{5}$ & Low risk & Low risk & Unclear & Unclear & Low risk & Low risk & Low risk \\
\hline Nishiyama et al ${ }^{15}$ & Low risk & Low risk & Unclear & Unclear & Low risk & Low risk & Low risk \\
\hline Komasawa et al ${ }^{16}$ & Low risk & Low risk & Unclear & Unclear & Low risk & Low risk & Low risk \\
\hline Reddy et al ${ }^{17}$ & Low risk & Unclear & Low risk & Low risk & Low risk & Low risk & Low risk \\
\hline
\end{tabular}


Table 4 Level of evidence and modified Jadad quality score

\begin{tabular}{|c|c|c|c|c|c|}
\hline \multirow[b]{2}{*}{ Reference } & \multirow[b]{2}{*}{ Level of evidence } & \multicolumn{4}{|c|}{ Modified Jadad Scale } \\
\hline & & Randomisation & Allocation concealment & Blinding & Withdrawals and dropouts \\
\hline Komasawa et $a l^{5}$ & 6 & 2 & 2 & 1 & 1 \\
\hline Komasawa et $a l^{16}$ & 6 & 2 & 2 & 1 & 1 \\
\hline Reddy et al ${ }^{17}$ & 6 & 2 & 1 & 2 & 1 \\
\hline
\end{tabular}

group and 71 patients in the control group. ${ }^{5} 16$ The incidence rate did not differ significantly between the prewarming group and the control group (RR 1.0; 95\% CI 0.14 to $6.90 ; \mathrm{p}=1.0$; figure 4$)$. There was low heterogeneity $\left(\mathrm{I}^{2}=0\right.$ and $\left.\mathrm{p}=1.00\right)$. We did not conduct the TSA and the Egger's regression test due to the sparse data. Only two trials reported on this outcome and no formal assessment for publication bias was made.

\section{Publication bias}

No publication bias was indicated among the included studies in both sealing pressure and first-attempt insertion success rate as illustrated by Egger's regression test $(p=0.7372$ for sealing pressure and $p=0.1378$ for firstattempt insertion success rate), respectively. Funnel plots were shown in figure 5 .
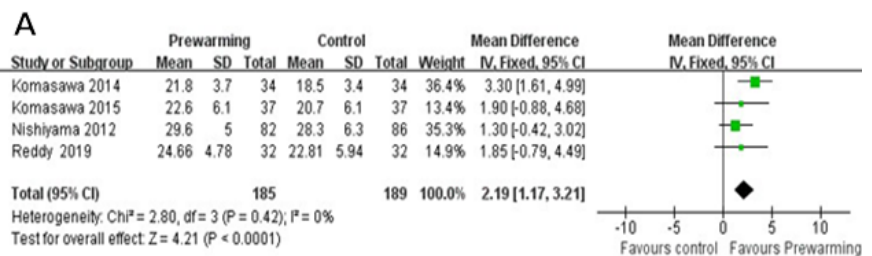

B

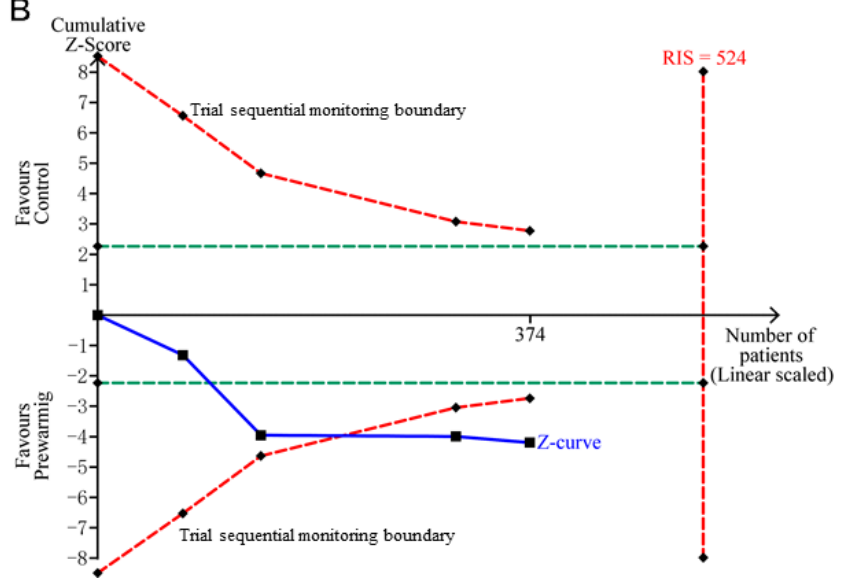

Figure 2 Forest plots and trial sequential analysis (TSA) of prewarming versus control on sealing pressure immediately after successful ventilation. (A) Forest plot for sealing pressure. (B) Mean difference and variance were defined by selecting the 'Low-bias Based' option in the TSA viewer (mean difference 2.19; variance 50.55). The continuous blue line represents the $Z$ line (cumulative effect size), red dashed lines represent the trial sequential monitoring boundaries, and required information size (RIS, the estimated optimal sample size adjusted to sample size and repeated analysis). The green dashed lines represent the conventional Cls.

\section{DISCUSSION}

\section{Main findings}

Our systematic review and meta-analysis revealed that prewarming i-gel laryngeal mask provides better mechanical ventilation efficiency with a higher sealing pressure immediately after successful ventilation compared with keeping it at room temperature. However, the superiority of the first insertion success rate and the incidence of the postoperative throat pain in the prewarming group was not different.

Sealing pressure is considered a measure of successful placement, adequate performance, and is a useful comparator between supraglottic airway devices. ${ }^{6} 27$ Sealing pressure is considered the most important determinant of the safety and efficacy of

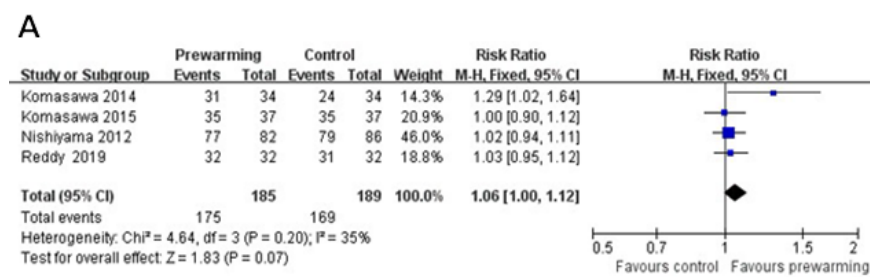

B

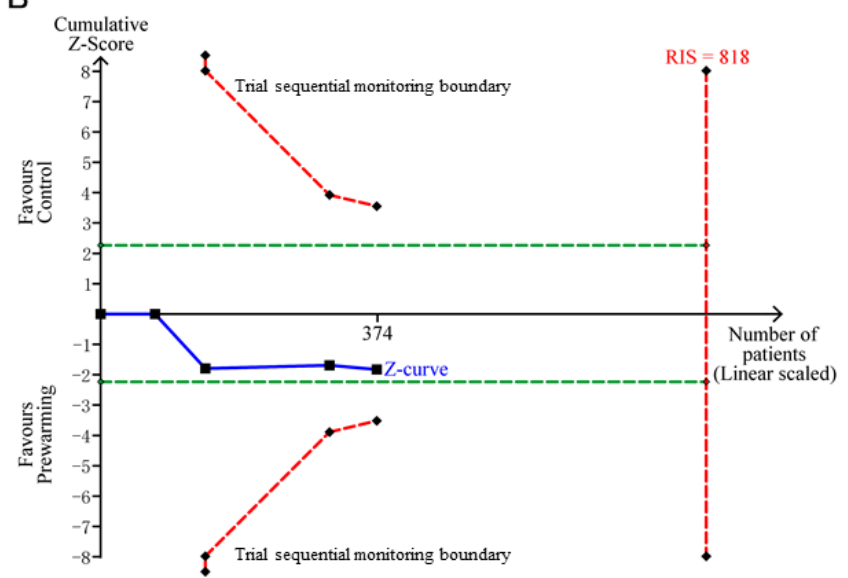

Figure 3 Forest plot and trial sequential analysis (TSA) of prewarming versus control on first-attempt insertion success rate. (A) Forest plot for first-attempt insertion success rate. (B) TSA for a relative risk improvement of $20 \%$. The continuous blue line represents the $Z$ line (cumulative effect size), red dashed lines represent the trial sequential monitoring boundaries, and required information size (RIS, the estimated optimal sample size adjusted to sample size and repeated analysis). The green dashed lines represent the conventional Cls. 


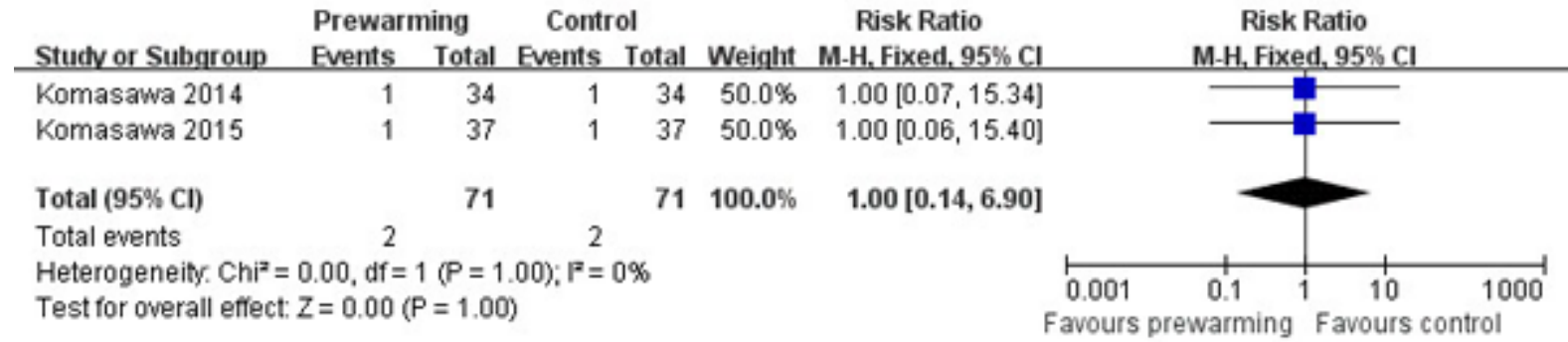

Figure 4 Forest plot for the incidence of postoperative pharyngeal pain.

any supraglottic airway device. ${ }^{28}{ }^{29}$ It is particularly useful in the efficiency of ventilation and airway protection prevention of aspiration. Higher pharyngeal sealing pressure provides better mechanical ventilation efficiency particularly during lithotomy position, patients with obesity and pneumoperitoneum. ${ }^{30-35}$ Some authors have found that the sealing pressure of i-gel laryngeal mask appears to improve over time, suggesting that i-gel laryngeal mask forms a more efficient seal around the larynx after warming to body temperature. ${ }^{9-11}$ Prewarming i-gel laryngeal mask to $42^{\circ} \mathrm{C}$ would enable the cuff to fit the pharyngeal structure more quickly than if it was stored at room temperature. ${ }^{5}$ But Dingley et $a l^{14}$ evaluated the properties of i-gel cuff over clinical temperature ranges and found that there was a minimal decrease in hardness and resilience with warming.

In clinical practice, whether the i-gel cuff will soften as it reaches body temperature after insertion remains controversial. Our meta-analysis found the sealing pressure of the prewarmed i-gel laryngeal mask was significantly higher than the control group. Martin $e t a l^{36}$ revealed that all i-gel mask has a significant temperature-dependent increase in volume and weight as well as a significant decrease in density. These results represent a new approach to explain how the prewarmed i-gel laryngeal mask improves its sealing pressure over time after insertion.

A

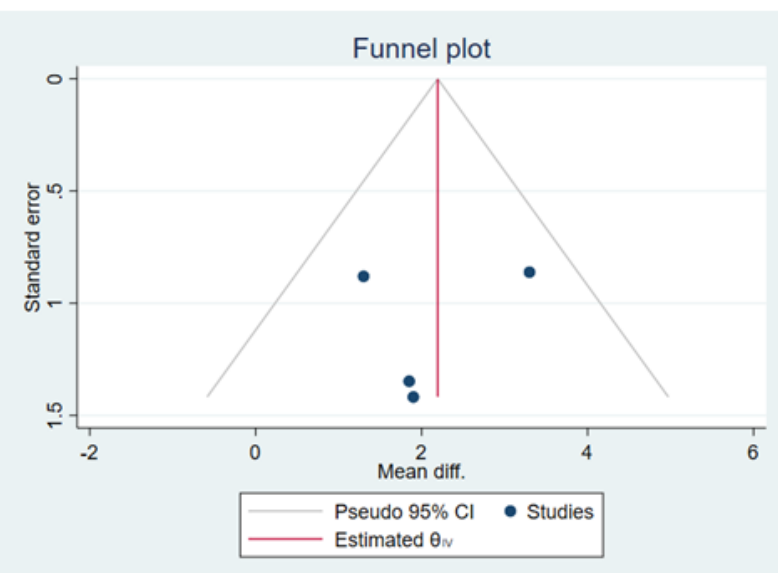

Komosawa et a $a \tilde{l}$ found that first insertion success rate was significantly higher when i-gel laryngeal mask was warmed to $42^{\circ} \mathrm{C}$ compared with it preserved at the room temperature, and they believe that the higher insertion success rate is a benefit of prewarming i-gel laryngeal mask. However, our result showed that first insertion success rate was not different in the prewarming and the control groups. We believe that the insertion success rate was also influenced by the practicing anesthesiologist's familiarity with i-gel laryngeal mask.

No difference was found in the incidence of postoperative pharyngeal pain between the prewarming group and the control group, the possible reasons are as follows: first, only two articles were included in this meta-analysis, TSA also indicated further trials are required. So, the quality of the results may be limited by the sample size. Second, cuff pressure was associated with postoperative pharyngeal pain, low cuff pressures reduced the incidence of postoperative pharyngeal pain. ${ }^{37}$ I-gel laryngeal mask avoids the inflation of cuff, then reduces the possibility of mucosal injury from over inflation. Then it has a lower incidence of postoperative sore throat compared with cuffed supraglottic airway devices. Based on the lower incidence of postoperative sore throat and small sample size, there was no difference in the incidence of postoperative pharyngeal pain between the prewarming group and the control group.

B

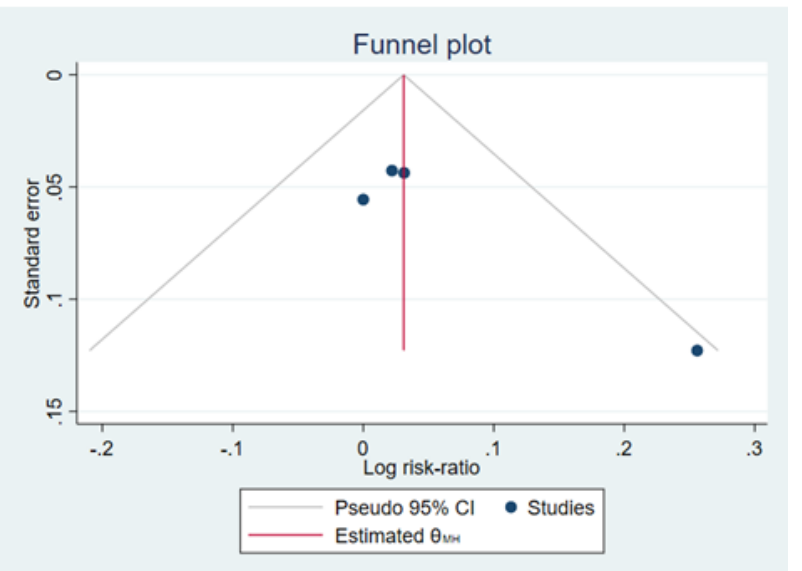

Figure 5 Funnel plot for publication bias of the included studies. (A) Distribution of studies included in sealing pressure. (B) Distribution of studies included in intubation rate. 


\section{Strengths and limitations of this study}

There are a few limitations in our meta-analysis. First, the practising anesthesiologist's familiarity with i-gel laryngeal mask plays an important role in the result of the first insertion success rate, but it was not mentioned in any of the included studies. Second, the prewarming temperature was different in the prewarming group, which may influence the result of the sealing pressure as i-gel laryngeal mask has a temperature-dependent volume increase. Third, analysis was based on a relatively small number of studies. Further studies examining larger number of participants in RCTs are required to confirm our findings.

\section{Implications and future research}

As the nature of thermoplastic material, prewarming i-gel laryngeal mask could enable the cuff to fit the pharyngeal structure more quickly, then provide a higher sealing pressure. Higher sealing pressure indicate the i-gel cuff fit the pharyngeal structure better and contact the adjacent soft tissues closer. It can protect the airway more efficiently, decrease the risk of regurgitation and pulmonary aspiration. Meanwhile, a few studies indicated the softened cuff after prewarming could probably improve the success intubation rate, reduce the risk for mucosal trauma and decrease the incidence of postoperative pharyngeal pain. ${ }^{51213}$ A relatively small number of studies were included in our meta-analysis, higher sealing pressure was the only beneficial effect of the prewarming treatment. However, we still hypothesised that prewarming i-gel laryngeal mask has beneficial effect on mechanical ventilation as the sample size increased. More RCT studies are needed to validate the efficacy of prewarming i-gel laryngeal mask for mechanical ventilation, particularly on the first-attempt insertion success rate and the postoperative pharyngeal pain in future.

\section{CONCLUSION}

Prewarming i-gel laryngeal mask provides a higher sealing pressure compared with keeping it at room temperature. But prewarming procedure did not increase the success rate of insertion, nor did it decrease the incidence of postoperative pharyngeal pain. More RCT studies are need to further confirm the beneficial effects of prewarming i-gel laryngeal mask.

Contributors JZ obtained funding. JZ, GC and LD designed and wrote the manuscript. JZ, LZ, JW and LD conducted the data collection analysis and edited the manuscript. JZ, GC, JW and LZ reviewed and gave the final approval for the manuscript. All authors read and approved the final manuscript.

Funding This study was supported by grants from the Sichuan Science and Technology Program (Grant No. 2019YJ0058). JZ is supported by Sichuan Science and Technology Program (Grant No. 2019YJ0058).

Competing interests None declared.

Patient consent for publication Not required.
Provenance and peer review Not commissioned; externally peer reviewed.

Data availability statement Data are available on reasonable request. The data that support the fndings of this study are available from the corresponding author, on reasonable request. All data relevant to the study are included in the article or uploaded as supplementary information.

Supplemental material This content has been supplied by the author(s). It has not been vetted by BMJ Publishing Group Limited (BMJ) and may not have been peer-reviewed. Any opinions or recommendations discussed are solely those of the author(s) and are not endorsed by BMJ. BMJ disclaims all liability and responsibility arising from any reliance placed on the content. Where the content includes any translated material, BMJ does not warrant the accuracy and reliability of the translations (including but not limited to local regulations, clinical guidelines, terminology, drug names and drug dosages), and is not responsible for any error and/or omissions arising from translation and adaptation or otherwise.

Open access This is an open access article distributed in accordance with the Creative Commons Attribution Non Commercial (CC BY-NC 4.0) license, which permits others to distribute, remix, adapt, build upon this work non-commercially, and license their derivative works on different terms, provided the original work is properly cited, appropriate credit is given, any changes made indicated, and the use is non-commercial. See: http://creativecommons.org/licenses/by-nc/4.0/.

ORCID iD

Jianqiao Zheng http://orcid.org/0000-0002-8091-1837

\section{REFERENCES}

1 de Montblanc J, Ruscio L, Mazoit JX, et al. A systematic review and meta-analysis of the i-gel(®) vs laryngeal mask airway in adults. Anaesthesia 2014;69:1151-62.

2 Maitra S, Baidya DK, Bhattacharjee S, et al. Evaluation of igel( $\left.{ }^{\mathrm{TM}}\right)$ airway in children: a meta-analysis. Paediatr Anaesth 2014;24:1072-9.

3 Levitan RM, Kinkle WC. Initial anatomic investigations of the I-gel airway: a novel supraglottic airway without inflatable cuff. Anaesthesia 2005;60:1022-6.

4 Russo SG, Cremer S, Eich C, et al. Magnetic resonance imaging study of the in vivo position of the extraglottic airway devices i-gel ${ }^{\mathrm{TM}}$ and LMA-Supreme ${ }^{T M}$ in anaesthetized human volunteers. $\mathrm{Br} J$ Anaesth 2012;109:996-1004.

5 Komasawa N, Nishihara I, Tatsumi S, et al. Prewarming of the i-gel facilitates successful insertion and ventilation efficacy with muscle relaxation: a randomized study. J Clin Anesth 2014;26:663-7.

6 Kumar CM, Van Zundert TC, Seet E, et al. Time to consider supraglottic airway device oropharyngeal leak pressure measurement more objectively. Acta Anaesthesiol Scand 2021;65:142-5.

7 Lopez-Gil M, Brimacombe J, Keller C. A comparison of four methods for assessing oropharyngeal leak pressure with the laryngeal mask airway (LMA) in paediatric patients. Paediatr Anaesth 2001;11:319-21.

8 Timmermann A, Cremer S, Eich C, et al. Prospective clinical and fiberoptic evaluation of the Supreme laryngeal mask airway. Anesthesiology 2009;110:262-5.

$9 \mathrm{Kim} \mathrm{M-S,} \mathrm{Lee} \mathrm{JH,} \mathrm{Han} \mathrm{SW,} \mathrm{et} \mathrm{al.} \mathrm{A} \mathrm{randomized} \mathrm{comparison} \mathrm{of} \mathrm{the}$ i-gel with the self-pressurized air- $Q$ intubating laryngeal airway in children. Paediatr Anaesth 2015;25:405-12.

$10 \mathrm{Kim} \mathrm{HJ}$, Lee K, Bai S, et al. Influence of head and neck position on ventilation using the air- $Q \mathbb{s} \mathrm{sp}$ airway in anaesthetized paralysed patients: a prospective randomized crossover study. $\mathrm{Br} J$ Anaesth 2017;118:452-7.

11 Janakiraman $C$, Chethan DB, Wilkes AR, et al. A randomised crossover trial comparing the i-gel supraglottic airway and classic laryngeal mask airway. Anaesthesia 2009;64:674-8.

$12 \mathrm{Kang} \mathrm{H}$, Kim DR, Jung YH, et al. Pre-warming the streamlined liner of the pharynx airway (SLIPA) improves fitting to the laryngeal structure: a randomized, double-blind study. BMC Anesthesiol 2015;15:167.

13 Geng G, Chen Y, Liu H. The effects of thermal softening of streamlined liner of pharyngeal airway before anesthesia induction on postoperative throat complications. Fudan Univ J Med Sci 2016;43:591-4.

14 Dingley J, Stephenson J, Allender V, et al. Changes in hardness and resilience of i-gel ${ }^{\mathrm{TM}}$ cuffs with temperature: a benchtop study. Anaesthesia 2018;73:856-62.

15 Nishiyama T, Kohno Y, Kim HJ, et al. The effects of prewarming the l-gel on fitting to laryngeal structure. Am J Emerg Med 2012;30:1756-9. 
16 Komasawa N, Nishihara I, Tatsumi S, et al. Does prewarming the i-gel supraglottic airway device fit the larynx better compared to keeping it at room temperature for non-paralysed, sedated patients: a randomised controlled trial. BMJ Open 2015;5:e006653.

17 Reddy AM, Varghese N, Herekar B, et al. Does prewarming of i-gel improve insertion and ventilation in anaesthetised and paralysed patients? A prospective, randomised, control trial. Saudi J Anaesth 2019;13:215-21.

18 Moher D, Shamseer L, Clarke M, et al. Preferred reporting items for systematic review and meta-analysis protocols (PRISMA-P) 2015 statement. Syst Rev 2015;4:1

19 Gheibi S, Mahmoodzadeh A, Kashfi K, et al. Data extraction from graphs using Adobe Photoshop: applications for meta-analyses. Int J Endocrinol Metab 2019;17:e95216.

20 Higgins JPT, Altman DG, Gøtzsche PC, et al. The Cochrane collaboration's tool for assessing risk of bias in randomised trials. BMJ 2011;343:d5928.

21 Jadad AR, Moore RA, Carroll D, et al. Assessing the quality of reports of randomized clinical trials: is blinding necessary? Control Clin Trials 1996;17:1-12.

22 Egger M, Davey Smith G, Schneider M, et al. Bias in meta-analysis detected by a simple, graphical test. BMJ 1997;315:629-34.

23 Sterne JA, Gavaghan D, Egger M. Publication and related bias in meta-analysis: power of statistical tests and prevalence in the literature. J Clin Epidemiol 2000;53:1119-29.

24 Casans-Francés R, Roberto-Alcácer AT, Gómez-Ríos MA, et al. The importance of trial sequential analysis in the evaluation of the results of a meta-analysis. Minerva Anestesiol 2019;85:342-3.

25 Wetterslev J, Jakobsen JC, Gluud C. Trial sequential analysis in systematic reviews with meta-analysis. BMC Med Res Methodol 2017;17:39.

26 Torlund K, Engstrøm J, Wetterslev J. User manual for trial sequential analysis (TSA). 2021. Copenhagen, Denmark: Copenhagen trial unit Centre for Clinical Intervention Research, 2011. http://www.ctu.dk/tsa
27 Keller C, Brimacombe JR, Keller K, et al. Comparison of four methods for assessing airway sealing pressure with the laryngeal mask airway in adult patients. $\mathrm{Br} J$ Anaesth 1999;82:286-7.

28 Kjaergard LL, Villumsen J, Gluud C. Reported methodologic quality and discrepancies between large and small randomized trials in meta-analyses. Ann Intern Med 2001;135:982-9.

29 Brimacombe J, Berry A, Brain Al. Optimal intracuff pressures with the laryngeal mask. Br J Anaesth 1996;77:295-6.

30 Beleña JM, Núñez M, Anta D, et al. Comparison of laryngeal mask airway Supreme and laryngeal mask airway Proseal with respect to oropharyngeal leak pressure during laparoscopic cholecystectomy: a randomised controlled trial. Eur J Anaesthesiol 2013;30:119-23.

31 Kim D-H, Park J-Y, Yu J, et al. Positive end-expiratory pressure increases arterial oxygenation in elderly patients undergoing urological surgery using laryngeal mask airway in lithotomy position. J Clin Monit Comput 2020;34:161-9.

32 Beleña JM, Ochoa EJ, Núñez M, et al. Role of laryngeal mask airway in laparoscopic cholecystectomy. World J Gastrointest Surg 2015;7:319-25

33 Lai C-J, Liu C-M, Wu C-Y, et al. I-Gel is a suitable alternative to endotracheal tubes in the laparoscopic pneumoperitoneum and Trendelenburg position. BMC Anesthesiol 2017;17:3.

34 Weber U, Oguz R, Potura LA, et al. Comparison of the i-gel and the LMA-Unique laryngeal mask airway in patients with mild to moderate obesity during elective short-term surgery. Anaesthesia 2011;66:481-7.

35 Prabha R, Raman R, Khan MP, et al. Comparison of I-gel for general anesthesia in obese and nonobese patients. Saudi $J$ Anaesth 2018;12:535-9.

36 Martin C, Piekarski F, Mutlak H, et al. Influence of temperature on volume, weight and density changes of i-gel masks. Anaesthesiol Intensive Ther 2020;52:119-25.

37 El-Boghdadly K, Bailey CR, Wiles MD. Postoperative sore throat: a systematic review. Anaesthesia 2016;71:706-17. 